\title{
DESIGN AND SYNTHESIS OF NOVEL 4-(4-FLUORO-3-METHYLPHENYL)-6-(SUBSTITUTED ARYL)-1,6-DIHYDROPYRIMIDIN-2-OL DERIVATIVES AS POTENT ANTI-INFLAMMATORY AND ANALGESIC AGENTS
}

\author{
MURALIDHARAN V, RAJA S*, ASHA DEEPTI \\ GITAM Institute of Pharmacy, GITAM (Deemed to be University), Visakhapatnam, Andhra Pradesh, India. Email: sraja61@gmail.com \\ Received: 29 November 2018, Revised and Accepted: 14 March 2019
}

ABSTRACT

Objective: Pyrimidine heterocycles possessing hydroxy group has a unique place in medicinal chemistry and also plays a key role in biological processes. In the biological functions at cellular level pyrimidine plays imperative roles which lead the researchers to design a variety of its derivatives. The aim of the present study was to synthesize the novel set of 4-(4-fluoro-3-methylphenyl)-6-(substituted aryl)-1,6-dihydropyrimidin-2-ol derivatives. These compounds were screened for their analgesic and anti-inflammatory activities.

Methods: A novel series of 4-(4-fluoro-3-methylphenyl)-6-(substituted aryl)-1,6-dihydro pyrimidin-2-ol derivatives were furnished in two steps starting from 4-fluoro-3-methyl acetophenone through chalcone formation. Human red blood cell membrane stabilization method and carrageenaninduced rat paw edema test were performed for screening in vitro and in vivo anti-inflammatory activity, respectively. Tail-flick technique was performed for screening analgesic activity.

Results: All the synthesized 4-(4-fluoro-3-methylphenyl)-6-(substituted aryl)-1,6-dihydro pyrimidin-2-ol derivatives were characterized by Fouriertransform infrared spectroscopy, ${ }^{1} \mathrm{H}$ nuclear magnetic resonance, mass spectroscopy, and bases of elemental analysis. The result of biological screening revealed that many of the new derivatives were endowed with improved anti-inflammatory and analgesic activities.

Conclusion: Nature of the substituent played a major role in anti-inflammatory and analgesic activities. The pyrimidine derivative with chlorophenyl substitution exhibited potent anti-inflammatory and analgesic activities. From the results, it was concluded that 6-(4-chlorophenyl)-4-(4-fluoro-3methyl phenyl)-1,6-dihydropyrimidin-2-ol was the most active compound.

Keywords: Pyrimidine, Chalcone, Anti-inflammatory, Analgesic, Carrageenan, Acetic acid.

(C) 2019 The Authors. Published by Innovare Academic Sciences Pvt Ltd. This is an open access article under the CC BY license (http://creativecommons. org/licenses/by/4. 0/) DOI: http://dx.doi.org/10.22159/ajpcr.2019.v12i4.31068

\section{INTRODUCTION}

Pyrimidine is ubiquitous in nature and found in a large group of biologically active compounds such as nucleic acids, vitamins, and coenzymes. They play a key role in the human physiological process. Pyrimidine [1] is a six-membered heterocycle with two nitrogen atoms situated in a 1,3-arrangement. The other name of pyrimidine is $m$-diazine or 1,3-diazine. Both nitrogen atoms in pyrimidines resemble pyridine nitrogen. The aromatic ring consists of a lone pair of electrons in the $s p^{2}$ hybrid orbital which belongs to the nitrogen atoms in its plane. These lone pairs are not needed for aromatic sextet; hence, they are basic in nature similar to pyridine. In the biological functions at cellular level pyrimidine plays a key role, which leads the researchers to design a variety of its derivatives. Pyrimidine heterocycles possessing hydroxyl group have a unique place in medicinal chemistry [2] and also plays a key role in biological processes [3]. The pharmacologically active drugs, namely barbituric acid and its several derivatives (e.g., Veranal) (Fig. 1) possess pyrimidine moiety in its main nucleus [4]. Pyrimidine derivatives provide a variety of biological activities such as cytotoxic [5], antimalarial [6], antioxidant [7], tyrosinase inhibitory [8], anti-inflammatory [9], cyclin-dependant kinase inhibitors [10], alopecia agent [11], and antibacterial [12].

The inflammation process involves a cascade that can be elicited by numerous stimuli (e.g. infectious agents, ischemia, and antigen-antibody interaction). Nonsteroidal anti-inflammatory drugs (NSAIDs) represent a heterogeneous family of pharmacologically potent compounds used to alleviate acute and chronic inflammation, pain, and fever. Almost two decades ago, steroidal drugs, namely prednisolone, dexamethasone, and betamthansone were considered to be the choicest and effective anti-inflammatory drugs. The serious and enormous adverse effects caused by either short-term or long-term usage of steroid therapy necessitated accelerated research toward the development of NSAIDs since the past two decades $[13,14]$. In the past decade, copious enhances have taken place in the understanding of pathogenesis, and as a result, momentous progress has been made and is still being made in the development of flawless NSAIDs [15]. Motivated by the aforesaid findings, and pursuing our studies on pyrimidine moiety, a new series of 4-(4-fluoro-3-methylphenyl)-6-(substituted aryl)-1,6dihydropyrimidin-2-ol derivatives were synthesized and tested for their anti-inflammatory and analgesic activities.

\section{METHODS}

Chemicals and reagents

The chemicals and reagents used in this work were obtained from various chemical units Avra, Sigma-Aldrich, SRL and SD Fine Chem. The solvents used were of LR grade and purified before their use. The silica gel $G$ used for analytical chromatography (thin-layer chromatography [TLC]) was obtained from E. Merck India Ltd. Solvent systems used were n-hexane:acetone (7:3).

\section{Instruments}

All the melting points were taken in open glass capillary and are uncorrected. ${ }^{1} \mathrm{H}$ nuclear magnetic resonance (NMR) spectra were taken on a Bruker ultra shield (400 MHz) NMR spectrometer in $\mathrm{CDCl}_{3}$ using tetramethylsilane [(CH3)4Si] as the internal standard. Chemical shift $(\delta)$ are expressed in ppm. Mass spectra were obtained on a JEOL-SX-102 instrument using electron impact ionization. All the IR spectra were recorded in $\mathrm{KBr}$ pellets on a Jasco Fourier-transform infrared (FT-IR) 


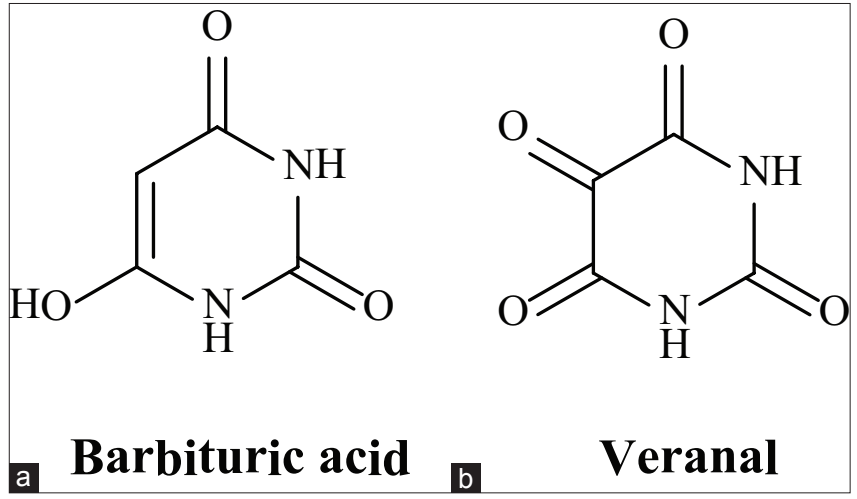

Fig. 1: (a and b) Hypnotic drugs with pyrimidine nucleus

410 spectrometer. Elemental analyses were performed on a Perkin Elmer model $240 \mathrm{c}$ analyzer and were within $\pm 0.4 \%$ of the theoretical values.

\section{Animals}

The animals were maintained in colony cages at $25 \pm 2^{\circ} \mathrm{C}$, relative humidity of $45-55 \%$, under a $12 \mathrm{~h}$ light and dark cycle and were fed standard animal feed [16]. All the animals were acclimatized for a week before use. The synthesized compounds were evaluated for their antiinflammatory and analgesic activities.

\section{Experimental work}

Preparation of (E)-1-(4-fluoro-3-methylphenyl)-3-(substituted aryl)prop-2-en-1-one (1a-1i)

The key intermediates (E)-1-(4-fluoro-3-methylphenyl)-3-(substituted aryl) prop-2-en-1-one (1a-1i) were prepared according to the reported literature [17]. The starting material 4-fluoro-3-methyl acetophenone $(0.02 \mathrm{~mol})$ was treated with aromatic aldehydes $(0.02 \mathrm{~mol})$ in the presence of catalytic amount of lithium hydroxide. Ethanol $(20 \mathrm{ml})$ was used as a solvent. The reaction mixture was kept for constant stirring using a multistage magnetic stirrer at room temperature until the solution turns turbid. The reaction was monitored by TLC (n-hexane:acetone - 7:3). Then, the reaction mixture was poured into crushed ice and neutralized with the help of dil.HCl. The precipitate was filtered under vacuum, washed with cold ethanol and distilled water. The obtained chalcones were purified by recrystallization and column chromatography.

1a (E)-1-(4-fluoro-3-methylphenyl)-3-(thiophen-2-yl) prop-2-en-1-one Greenish yellow crystals $\left(\right.$ EtOH), yield $=81 \%$; m.p. $101-103^{\circ} \mathrm{C}$. FTIR (KBr) cm-1: 1657 (C=0, chalcone), 1585 (C=C), 1149 (C-F), 2949 $\left(\mathrm{C}-\mathrm{CH}_{3}\right),{ }^{1} \mathrm{HNMR}\left(400 \mathrm{MHz}, \mathrm{CDCl}_{3} \delta \mathrm{ppm}\right): 2.358\left(\mathrm{~s}, 3 \mathrm{H}, \mathrm{CH}_{3}\right), 7.430(\mathrm{~d}$, $1 \mathrm{H}, \alpha-\mathrm{H}), 7.921(\mathrm{~d}, 1 \mathrm{H}, \beta-\mathrm{H}), 7.42(\mathrm{~s}, 1 \mathrm{H}, \mathrm{Ar}-\mathrm{H}), 7.32(\mathrm{~d}, 1 \mathrm{H}, \mathrm{Ar}-\mathrm{H}), 7.848$ (d, 1H, Ar-H), 7.86 (d, 1H, Ar-H), 7.433 (d, 1H, Ar-H), 7.972 (s, 1H, Ar-H). MS (EI) m/z: $247\left(\mathrm{M}^{+}\right)$. Anal. calcd for $\mathrm{C}_{14} \mathrm{H}_{11}$ FOS: C, 68.27; H, 4.50; F, $7.71 ; 0,6.50 ; \mathrm{S}, 13.02$.

1b (E)-3-(2-bromophenyl)-1-(4-fluoro-3-methylphenyl) prop-2-en-1-one Yellow crystals $(\mathrm{EtOH})$, yield $=76 \%$; m.p. $100-102^{\circ} \mathrm{C}$. FT-IR $(\mathrm{KBr})$ $\mathrm{cm}^{-1}: 1658$ (C=0, chalcone), $1588(\mathrm{C}=\mathrm{C}), 2924\left(\mathrm{C}-\mathrm{CH}_{3}\right), 1153(\mathrm{C}-\mathrm{F}), 752.9$ (C-Br). ${ }^{1} \mathrm{HNMR}\left(400 \mathrm{MHz}, \mathrm{CDCl}_{3} \delta \mathrm{ppm}\right): 2.384\left(\mathrm{~s}, 3 \mathrm{H},-\mathrm{CH}_{3}\right), 7.374(\mathrm{~d}, 1 \mathrm{H}$, $\alpha-\mathrm{H}), 8.155(\mathrm{~d}, 1 \mathrm{H}, \beta-\mathrm{H}), 7.119-7.93(\mathrm{~d}, 7 \mathrm{H}, \mathrm{Ar}-\mathrm{H})$. MS (EI) m/z: $319\left(\mathrm{M}^{+}\right)$. Anal. calcd for $\mathrm{C}_{16} \mathrm{H}_{12} \mathrm{BrFO}$ : C, 60.21; H, 3.79; F, 5.95; O, 5.01; Br, 25.04.

1c (E)-1-(4-fluoro-3-methylphenyl)-3-(2-nitrophenyl) prop-2-en-1-one Orange crystals $(\mathrm{EtOH})$, yield $=77 \%$; m.p. 94-96 ${ }^{\circ} \mathrm{C}$. FT-IR $(\mathrm{KBr}) \mathrm{cm}^{-1}$ : $1674\left(\mathrm{C}=\mathrm{O}\right.$, chalcone), 1513(C=C), $2878\left(\mathrm{C}-\mathrm{CH}_{3}\right), 1292(\mathrm{C}-\mathrm{F}), 1341$ (C-NO $) .{ }^{1} \mathrm{HNMR}\left(400 \mathrm{MHz}, \mathrm{CDCl}_{3} \delta \mathrm{ppm}\right): 2.39\left(\mathrm{~s}, 3 \mathrm{H}, \mathrm{CH}_{3}\right), 7.86(\mathrm{~d}, 1 \mathrm{H}$, $\alpha-\mathrm{H}), 8.09(\mathrm{~d}, 1 \mathrm{H}, \beta-\mathrm{H}), 7.585(\mathrm{~s}, 1 \mathrm{H}, \mathrm{Ar}-\mathrm{H}), 7.173(\mathrm{~d}, 1 \mathrm{H}, \mathrm{Ar}-\mathrm{H}), 7.71(\mathrm{~d}$, $1 \mathrm{H}, \mathrm{Ar}-\mathrm{H}) 8.15$ (s, 1H, Ar-H), $8.112(\mathrm{~d}, 1 \mathrm{H}, \mathrm{Ar}-\mathrm{H}), 7.582(\mathrm{~d}, 1 \mathrm{H}, \mathrm{Ar}-\mathrm{H})$, 7.928 (d, Ar-H). MS (EI) m/z: $284\left(\mathrm{M}^{+}\right)$. Anal. calcd for $\mathrm{C}_{16} \mathrm{H}_{12} \mathrm{FNO}_{3}$ : C, 67.36; H, 4.24; F, 6.66; N, 4.91;0,16.83. 1d (E)-1-(4-fluoro-3-methylphenyl)-3-(4-hydroxy-3-methoxy-5-nitro phenyl)prop-2-en-1-one

Pale yellow crystals $(\mathrm{EtOH})$, yield $=78 \%$; m.p. $91-93^{\circ} \mathrm{C}$. FT-IR $(\mathrm{KBr}) \mathrm{cm}^{-1}$ : 1684 (C=0, chalcone), 1546 (C=C), $2944\left(\mathrm{C}-\mathrm{CH}_{3}\right), 1103$ (C-F), 1230 $\left(\mathrm{C}-\mathrm{OCH}_{3}\right), 1366\left(\mathrm{NO}_{2}\right), 3200(\mathrm{OH}) .{ }^{1} \mathrm{HNMR}\left(400 \mathrm{MHz}, \mathrm{CDCl}_{3} \delta \mathrm{ppm}\right)$ $\mathrm{CH}_{3} 2.186\left(\mathrm{~s}, 3 \mathrm{H}, \mathrm{CH}_{3}\right), 1.727(\mathrm{~s}, 1 \mathrm{H}, \mathrm{OH}) 4.043\left(\mathrm{~s}, 3 \mathrm{H},-\mathrm{OCH}_{3}\right), 7.664(\mathrm{~d}, 1 \mathrm{H}$, $\alpha-\mathrm{H}), 8.245$ (d, $1 \mathrm{H}, \beta-\mathrm{H}$ ), 7.285-8.245(m, 5H, Ar-H). MS (EI) m/z: 331.09 $\left(\mathrm{M}^{+}\right)$. Anal. calcd for $\mathrm{C}_{17} \mathrm{H}_{14} \mathrm{NO}_{5}$ : C, 61.63; H, 4.26; F, 5.73; N, 4.23; O, 24.15 .

1e (E)-3-(3,4-dimethoxyphenyl)-1-(4-fluoro-3-methylphenyl)prop-2en-1-one

Lemon yellow crystals $\left(\right.$ EtOH), yield $=81 \%$; m.p. $85-87^{\circ} \mathrm{C}$. FT-IR ( $\mathrm{KBr}) \mathrm{cm}^{-1}: 1656$ (C=0, chalcone), 1583(C=C), $2930\left(\mathrm{C}^{-\mathrm{CH}_{3}}\right), 1254(\mathrm{C}-\mathrm{F})$, $1142\left(\mathrm{C}-\mathrm{OCH}_{3}\right) .{ }^{1} \mathrm{HNMR}\left(400 \mathrm{MHz}, \mathrm{CDCl}_{3} \delta \mathrm{ppm}\right): 2.35\left(\mathrm{~s}, 3 \mathrm{H}, \mathrm{CH}_{3}\right)$, $3.936\left(\mathrm{~d}, 6 \mathrm{H},-\mathrm{OCH}_{3}\right), 7.739(\mathrm{~d}, 1 \mathrm{H}, \alpha-\mathrm{H}), 7.905(\mathrm{~d}, 1 \mathrm{H}, \beta-\mathrm{H}), 7.38(\mathrm{~s}, 1 \mathrm{H}$, $\mathrm{Ar}-\mathrm{H}), 7.25(\mathrm{~d}, 1 \mathrm{H}, \mathrm{Ar}-\mathrm{H}), 7.864(\mathrm{~d}, 1 \mathrm{H}, \mathrm{Ar}-\mathrm{H}), 7.23(\mathrm{~s}, 1 \mathrm{H}, \mathrm{Ar}-\mathrm{H}), 7.080$ (d, 1H, Ar-H), 6.913 (d, 1H, Ar-H). MS (EI) m/z: $301\left(\mathrm{M}^{+}\right)$. Anal. calcd for $\mathrm{C}_{18} \mathrm{H}_{17} \mathrm{FO}_{3}: \mathrm{C}, 71.99 ; \mathrm{H}, 5.71 ; \mathrm{F}, 6.33 ; 0,15.98$.

$1 \mathrm{f}$ (E)-3-(4-(dimethylamino)phenyl)-1-(4-fluoro-3-methylphenyl) prop -2-en-1-one

Bright red crystals (EtOH), yield $=83 \%$; m.p. $93-95^{\circ} \mathrm{C}$. FT-IR $(\mathrm{KBr}) \mathrm{cm}^{-1}$ : 1651 (C=0, chalcone), 1593(C=C), $2923\left(\mathrm{C}-\mathrm{CH}_{3}\right), 1243(\mathrm{C}-\mathrm{F}) .{ }^{1} \mathrm{HNMR}(400$ $\left.\mathrm{MHz}, \mathrm{CDCl}_{3} \delta \mathrm{ppm}\right): 2.37\left(\mathrm{~s}, 3 \mathrm{H}, \mathrm{CH}_{3}\right), 3.06\left(\mathrm{~s}, 6 \mathrm{H}, \mathrm{N}\left(\mathrm{CH}_{3}\right), 7.913(\mathrm{~d}, 1 \mathrm{H}\right.$, $\alpha-\mathrm{H}), 7.577$ (d, 1H, $\beta-\mathrm{H}), 7.34$ (s, 1H, Ar-H), 7.03 (d, 1H, Ar-H), 7.849 (d, $1 \mathrm{H}, \mathrm{Ar}-\mathrm{H}), 7.78$ (s, 2H, Ar-H), 6.734 (d, 2H, Ar-H). MS (EI) m/z: $284\left(\mathrm{M}^{+}\right)$. Anal. calcd for $\mathrm{C}_{18} \mathrm{H}_{18}$ FNO: C, 76.30; $\mathrm{H}, 6.40 ; \mathrm{F}, 6.71 ; \mathrm{O}, 5.65 ; \mathrm{N}, 4.94$.

1g (E)-3-(4-chlorophenyl)-1-(4-fluoro-3-methylphenyl) prop-2-en-1-one Lemon yellow crystals $(\mathrm{EtOH})$, yield $=77 \%$; m.p. $101-103^{\circ} \mathrm{C}$. FT-IR $(\mathrm{KBr}) \mathrm{cm}^{-1}: 1663\left(\mathrm{C}=0\right.$, chalcone), 1591(C=C), $2960\left(\mathrm{C}-\mathrm{CH}_{3}\right), 1243(\mathrm{C}-$ F), 819 (C-Cl). ${ }^{1} \mathrm{HNMR}\left(400 \mathrm{MHz}, \mathrm{CDCl}_{3} \delta \mathrm{ppm}\right): 2,38\left(\mathrm{~s}, 3 \mathrm{H}, \mathrm{CH}_{3}\right), 7,591$ (d, $1 \mathrm{H}, \alpha-\mathrm{H}), 7,93(\mathrm{~d}, 1 \mathrm{H}, \beta-\mathrm{H}), 7,58(\mathrm{~s}, 1 \mathrm{H}, \mathrm{Ar}-\mathrm{H}), 7,433(\mathrm{~d}, 1 \mathrm{H}, \mathrm{Ar}-\mathrm{H}), 7,61$ (d, 1H, Ar-H), 7,60 (s, 1H, Ar-H), 7,75 (d, 1H, Ar-H), 7,47 (d, 1H, Ar-H). MS (EI) m/z: $275\left(\mathrm{M}^{+}\right)$. Anal. calcd for $\mathrm{C}_{16} \mathrm{H}_{12} \mathrm{ClFO}$ : C, 69.95; H, 4.40; $\mathrm{Cl}$, $12.91 ; \mathrm{F}, 6.92 ; 0,5.82$.

$1 \mathrm{~h}$ (E)-3-(anthracen-9-yl)-1-(4-fluoro-3-methylphenyl) prop-2-en-1-one Orange crystals $(\mathrm{EtOH})$, yield $=79 \%$; m.p. $91-93^{\circ} \mathrm{C}$. FT-IR $(\mathrm{KBr}) \mathrm{cm}^{-1}$ : 1658 (C=0, chalcone), $1591(\mathrm{C}=\mathrm{C}), 2924\left(\mathrm{C}-\mathrm{CH}_{3}\right), 1261$ (C-F). ${ }^{1} \mathrm{HNMR}(400$ $\left.\mathrm{MHz}, \mathrm{CDCl}_{3} \delta \mathrm{ppm}\right): 2.376\left(\mathrm{~s}, 3 \mathrm{H}, \mathrm{CH}_{3}\right), 7.558(\mathrm{~d}, 1 \mathrm{H}, \alpha-\mathrm{H}), 7.964$ (d, $1 \mathrm{H}, \beta-\mathrm{H}), 7.602$ (s, 1H, Ar-H), 7.964 (d, 1H, Ar-H), 7.37 (d, 1H, Ar-H), 7.43-9.03 (m, 9H, Anthracene-H). MS (EI) m/z: $340\left(\mathrm{M}^{+}\right)$. Anal. calcd for $\mathrm{C}_{24} \mathrm{H}_{17}$ FO: C, 84.68; H, 5.03; F, 5.58; 0, 4.70 .

1i (E)-3-(5-bromo-2-hydroxy-3-methoxyphenyl)-1-(4-fluoro-3-methyl phenyl)prop-2-en-1- one

Orange crystals $(\mathrm{EtOH})$, yield $=69 \%$; m.p. $93-95^{\circ} \mathrm{C}$, FT-IR $(\mathrm{KBr}) \mathrm{cm}^{-1}$ : $1656\left(\mathrm{C}=\mathrm{O}\right.$, chalcone), 1581(C=C), $2923\left(\mathrm{C}-\mathrm{CH}_{3}\right), 1195$ (C-F), C-OH (3243), C-OCH 3 1255), C-Br (705): ${ }^{1} \mathrm{HNMR}\left(400 \mathrm{MHz}, \mathrm{CDCl}_{3}, \delta \mathrm{ppm}\right)$ : $1,617\left(\mathrm{~s}, 3 \mathrm{H}, \mathrm{CH}_{3}\right.$ ) , 7,34 (d, $\left.1 \mathrm{H}, \alpha-\mathrm{H}\right), 9,879(\mathrm{~s}, 1 \mathrm{H}, \beta-\mathrm{H}), 7,203(\mathrm{~d}, 5 \mathrm{H}$, Ar-H), 7,285(s, 1H, OH), 3,943(s, 3H, C-OCH ${ }_{3}$ : MS (EI) m/z: $365\left(\mathrm{M}^{+}\right)$: Anal. calcd for $\mathrm{C}_{17} \mathrm{H}_{14} \mathrm{FBrO}_{3}$ : C, 55.91; $\mathrm{H}, 3.83 ; \mathrm{Br}, 21.88 ; \mathrm{F}, 5.20 ; 0,13.14$.

Synthesis of 4-(4-fluoro-3-methylphenyl)-6-(substituted aryl)1,6-dihydropyrimidin-2-ol. (2a-2i)

The mixture of chalcones $(0.002 \mathrm{~mol})$, urea $(0.002 \mathrm{~mol})$, and $5 \mathrm{ml}$ of $\mathrm{HCl}$ in absolute ethanol $(20 \mathrm{ml})$ was refluxed on a water bath for 6-8 $\mathrm{h}$. The reaction was monitored by TLC. After completion of the reaction, $40 \%$ ammonia was added to neutralize the reaction mixture. The reaction mixture was kept in the refrigerator for $2 \mathrm{~h}$. The precipitate obtained was filtered under vacuum and recrystallized using ethanol to obtain 4-(4-fluoro-3-methylphenyl)-6-(substituted aryl)-1,6dihydropyrimidin-2-ol derivatives.

2a 4-(4-fluoro-3-methylphenyl)-6-(thiophen-2-yl)-1,6-dihydropyrimidin2-ol.

Yield 75\%, m.p. $168^{\circ} \mathrm{C}$, FT-IR (KBr) cm${ }^{-1}: 1041.13$ (C-F); 2918.24 (C-CH ${ }_{3} \mathrm{Str}$ ); 3073.70 (Ar C-H str); 1667.52 (C=N str); 1585.11 ( $\mathrm{Ar} \mathrm{C}=\mathrm{C}$ ); 
3449.10 (NH str); ${ }^{1} \mathrm{HNMR}\left(400 \mathrm{MHz}, \mathrm{CDCl}_{3}, \delta \mathrm{ppm}\right) 2.388\left(\mathrm{~s}, 3 \mathrm{H}, \mathrm{CH}_{3}\right)$; $1.593(\mathrm{~m}, 1 \mathrm{H}, \mathrm{N}-\mathrm{H}) ; 2.194$ (d, 1H, C' '-H pyrimidine); $7.112\left(\mathrm{~d}, 1 \mathrm{H}, \mathrm{C5}^{\prime}-\mathrm{H}\right.$ pyrimidine); $7.134\left(\mathrm{~m}, 2 \mathrm{H}, \mathrm{C}_{5}\right.$ and $\left.\mathrm{C}_{2}-\mathrm{H}\right) ; 7.125\left(\mathrm{~d}, \mathrm{~J}=5.2 \mathrm{~Hz}, 1 \mathrm{H}, \mathrm{C}_{6}-\mathrm{H}\right)$; 7.304 (d, J=7.6Hz, 1H, C' $-\mathrm{H}$ ); 7.397 (d, J= 3.7 1H, C ' - -H): MS (EI) m/z: $288\left(\mathrm{M}^{+}\right)$. Anal. calcd for $\mathrm{C}_{15} \mathrm{H}_{13} \mathrm{FN}_{2} \mathrm{OS}$; C, 62.48; $\mathrm{H}, 4.54 ; \mathrm{F}, 6.59 ; \mathrm{N}, 9.72$; $0,5.55 ;$ S, 11.12 .

2b 4-(4-fluoro-3-methylphenyl)-6-(2-bromophenyl)-1,6-dihydropyrim idin-2-ol

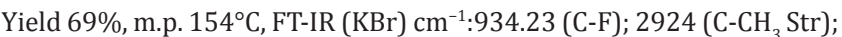
753 (C-Br); 3050 (Ar C-H str); 1662 (C=N str); 1588 (Ar C=C); 3624 (OH): ${ }^{1} \mathrm{HNMR}\left(400 \mathrm{MHz}, \mathrm{CDCl}_{3}, \delta \mathrm{ppm}\right) 2.388\left(\mathrm{~s}, 3 \mathrm{H}, \mathrm{CH}_{3}\right) ; 1.640(\mathrm{~s}, 1 \mathrm{H}, \mathrm{N}-\mathrm{H})$; 2.192 (d, J=2Hz, 1H, C - - $-\mathrm{H}$ pyrimidine); 7.119 (d, $1 \mathrm{H}, \mathrm{C}_{5}-\mathrm{H}$ pyrimidine); $7.163\left(\mathrm{~m}, 2 \mathrm{H}, \mathrm{C}_{5}\right.$ and $\left.\mathrm{C}_{2}-\mathrm{H}\right) ; 7.425\left(\mathrm{~m}, 1 \mathrm{H}, \mathrm{C}_{6}-\mathrm{H}\right) ; 7.141-8.153(\mathrm{~m}, 4 \mathrm{H}$, Ar-H): MS (EI) m/z: $360\left(M^{+}\right.$). Anal. calcd for $\mathrm{C}_{17} \mathrm{H}_{14} \mathrm{BrFN}_{2} \mathrm{O} ; \mathrm{C}, 56.53 ; \mathrm{H}$, 3.91; $\mathrm{Br}, 22.12 ; \mathrm{F}, 5.26 ; \mathrm{N}, 7.76 ; 0,4.43$.

2c 4-(4-fluoro-3-methylphenyl)-6-(2-nitrophenyl)-1,6-dihydropyrimi dine-2-ol

Yield 79\%, m.p. $162^{\circ} \mathrm{C}$, FT-IR (KBr) cm ${ }^{-1}: 1029.45$ (C-F); 2927.46 (C-CH ${ }_{3}$ Str); 3073.32 (ArC-H str); 1669.54 (C=N str); 1511 ( $\mathrm{Ar} \mathrm{C}=\mathrm{C}$ ); 1340 $\left(\mathrm{NO}_{2}\right)$ : ${ }^{1} \mathrm{HNMR}\left(400 \mathrm{MHz}, \mathrm{CDCl}_{3} \delta \mathrm{ppm}\right) 2.394\left(\mathrm{~s}, 3 \mathrm{H}, \mathrm{CH}_{3}\right) ; 2.193(\mathrm{~m}$, $1 \mathrm{H}, \mathrm{N}-\mathrm{H}) ; 3.519\left(\mathrm{~d}, \mathrm{~J}=1.6 \mathrm{~Hz}, 1 \mathrm{H}, \mathrm{C}_{6}-\mathrm{H}\right.$ pyrimidine); $7.173\left(\mathrm{~d}, 1 \mathrm{H}, \mathrm{C}_{5}-\mathrm{H}\right.$ pyrimidine); $7.269\left(\mathrm{~m}, 1 \mathrm{H}, \mathrm{C}_{5}-\mathrm{H}\right) ; 7.308\left(\mathrm{~m}, 1 \mathrm{H}, \mathrm{C}_{5}-\mathrm{H}\right) ; 7.544(\mathrm{~m}, 1 \mathrm{H}$, $\left.\mathrm{C}_{6}-\mathrm{H}\right) ; 7.51\left(\mathrm{~m}, 1 \mathrm{H}, \mathrm{C}_{4^{\prime \prime}}-\mathrm{H}\right) ; 7.94\left(\mathrm{~m}, 1 \mathrm{H}, \mathrm{C}_{3^{\prime \prime}}-\mathrm{H}\right) ; 7.867\left(\mathrm{~m}, 1 \mathrm{H}, \mathrm{C}_{6^{\prime \prime}}-\mathrm{H}\right)$; $8.152\left(\mathrm{~m}, 1 \mathrm{H}, \mathrm{C}_{3}{ }^{\prime \prime}-\mathrm{H}\right)$ : MS (EI) m/z: $327\left(\mathrm{M}^{+}\right)$. Anal. calcd for $\mathrm{C}_{17} \mathrm{H}_{14} \mathrm{FN}_{3} \mathrm{O}_{3}$; C, 62.38; H, 4.13; F, 5.08; N, 12.84; 0, 14.66 .

2d 4-(4-fluoro-3-methylphenyl)-6-(4-hydroxy-3-methoxy-5-nitrophen yl)-1,6-dihydro pyrimidine-2-ol

Yield 71\%, m.p. $172^{\circ}$ C, FT-IR (KBr) cm ${ }^{-1}: 1044.45$ (C-F); $2921.34\left(C^{-}-\mathrm{CH}_{3}\right.$ Str); 3076.17 (Ar C-H str); 1610.70 (C=N str); 1546 (Ar C=C); 3403.75 (NH str); $3735(\mathrm{OH}) ; 1463\left(\mathrm{NO}_{2}\right) ; 1269\left(\mathrm{OCH}_{3}\right):{ }^{1} \mathrm{HNMR}\left(400 \mathrm{MHz}, \mathrm{CDCl}_{3}\right.$ $\delta$ ppm) $2.193\left(\mathrm{~s}, 3 \mathrm{H}, \mathrm{CH}_{3}\right) ; 1.606(\mathrm{~s}, 1 \mathrm{H}, \mathrm{O}-\mathrm{H}) ; 11.273(\mathrm{~m}, 1 \mathrm{H}, \mathrm{N}-\mathrm{H})$; 3.518 (d, J=4.8Hz, 1H, C' $-\mathrm{H}$ pyrimidine); $4.051\left(\mathrm{~s}, 3 \mathrm{H}, \mathrm{OCH}_{3}\right) ; 7.128$ $\left(\mathrm{m}, 3 \mathrm{H}, \mathrm{C}_{2}, \mathrm{C}_{5}, \mathrm{C}_{5^{5}}-\mathrm{H}\right) ; 7.673\left(\mathrm{~m}, 1 \mathrm{H}, \mathrm{C}_{6}-\mathrm{H}\right) ; 8.253\left(\mathrm{~m}, 2 \mathrm{H}, \mathrm{C}_{2^{\prime \prime}}-\mathrm{H}\right.$ and C $\left.{ }_{6}{ }^{\prime \prime}-\mathrm{H}\right): \mathrm{MS}$ (EI) $\mathrm{m} / \mathrm{z}: 373\left(\mathrm{M}^{+}\right)$. Anal. calcd for $\mathrm{C}_{17} \mathrm{H}_{14} \mathrm{FN}_{3} \mathrm{O}_{3} ; \mathrm{C}, 62.38 ; \mathrm{H}, 4.13$; F,5.08; $\mathrm{N}, 12.84 ; 0,14.66$

2e 6-(3,4-dimethoxyphenyl)-4-(4-fluoro-3-methylphenyl)-1,6-dihydro pyrimidine-2-ol

Yield 66\%, m.p. $144^{\circ} \mathrm{C}$, FT-IR (KBr) cm ${ }^{-1}: 1023.84$ (C-F); $2932.06\left({\mathrm{C}-\mathrm{CH}_{3}}\right.$ Str); 3075.07 (Ar C-H str); 1657.22 (C=N str); 1583 (Ar C=C); 3611 (OH); 1256 and $1142\left(\mathrm{OCH}_{3}\right)$ : ${ }^{1} \mathrm{HNMR}\left(400 \mathrm{MHz}, \mathrm{CDCl}_{3} \delta \mathrm{ppm}\right) 2.387$ (s, $\left.3 \mathrm{H}, \mathrm{CH}_{3}\right) ; 1.641(\mathrm{~s}, 1 \mathrm{H}, \mathrm{O}-\mathrm{H}) ; 2.192(\mathrm{~m}, 1 \mathrm{H}, \mathrm{N}-\mathrm{H}) ; 3.515$ (d, J=4.8Hz, $1 \mathrm{H}, \mathrm{C}_{6}-\mathrm{H}$ pyrimidine); $3.981\left(\mathrm{~d}, \mathrm{~J}=8 \mathrm{~Hz}, 6 \mathrm{H}, \mathrm{OCH}_{3}\right) ; 6.941(\mathrm{~d}, \mathrm{~J}=8.4 \mathrm{~Hz}, 1 \mathrm{H}$, $\mathrm{C}_{5}-\mathrm{H}$ pyrimidine); $7.184\left(\mathrm{~m}, 1 \mathrm{H}, \mathrm{C}_{2}-\mathrm{H}\right) ; 7.154\left(\mathrm{~m}, 1 \mathrm{H}, \mathrm{C}_{5}-\mathrm{H}\right) ; 7.394(\mathrm{~m}$, 1H, C $-\mathrm{H}) ; 7.761-7.924\left(\mathrm{~m}, 3 \mathrm{H}, \mathrm{C}_{2^{\prime \prime}}-\mathrm{H}, \mathrm{C}_{5^{\prime \prime}}-\mathrm{H}\right.$ and $\left.\mathrm{C}_{6^{\prime \prime}}-\mathrm{H}\right)$ : MS (EI) m/z: 342 $\left(\mathrm{M}^{+}\right)$. Anal. calcd for $\mathrm{C}_{19} \mathrm{H}_{19} \mathrm{FN}_{2} \mathrm{O}_{3} ; \mathrm{C}, 66.66 ; \mathrm{H}, 5.59 ; \mathrm{F}, 5.55 ; \mathrm{N}, 8.18 ; \mathrm{O}$, 14.02

2f 6-(4-(dimethylamino)phenyl)-4-(4-fluoro-3-methylphenyl)-1,6-dihy dropyrimidine-2-ol

Yield 82\%, m.p. $158^{\circ}$ C, FT-IR (KBr) cm ${ }^{-1}: 1053.59$ (C-F); 2919.47 (C-CH Str); 3434.74 (Ar NH str); 1648.76 (C=N str); 1594 (Ar C=C); 1299.42 (C-N str) ${ }^{1} \mathrm{HNMR}\left(400 \mathrm{MHz}, \mathrm{CDCl}_{3} \delta \mathrm{ppm}\right) 2.378\left(\mathrm{~s}, 3 \mathrm{H}, \mathrm{CH}_{3}\right) ; 1.597(\mathrm{~s}, 1 \mathrm{H}$, O-H); $2.194(\mathrm{~m}, 1 \mathrm{H}, \mathrm{N}-\mathrm{H}) ; 3.075\left(\mathrm{~s}, 6 \mathrm{H}, \mathrm{N}-\left(\mathrm{CH}_{3}\right)_{2}\right) ; 3.521$ (d, J=5.6Hz, $1 \mathrm{H}, \mathrm{C}_{6}-\mathrm{H}$ pyrimidine); $6.734\left(\mathrm{~d}, \mathrm{~J}=5.2 \mathrm{~Hz}, 1 \mathrm{H}, \mathrm{C}_{5}-\mathrm{H}\right.$ pyrimidine); 7.133 (m, 1H, C $-\mathrm{H}) ; 7.342\left(\mathrm{~m}, 1 \mathrm{H}, \mathrm{C}_{5}-\mathrm{H}\right) ; 7.586\left(\mathrm{~m}, 1 \mathrm{H}, \mathrm{C}_{6}-\mathrm{H}\right) ; 7.544-7.913$ (m, $4 \mathrm{H}, \mathrm{C}_{2^{\prime \prime}}-\mathrm{H}, \mathrm{C}_{5^{\prime \prime}}-\mathrm{H}$ and $\left.\mathrm{C}_{6^{\prime \prime}}-\mathrm{H}\right)$ : MS (EI) m/z: $325\left(\mathrm{M}^{+}\right)$. Anal. calcd for $\mathrm{C}_{19} \mathrm{H}_{20} \mathrm{FN}_{3} \mathrm{O}_{4} ; \mathrm{C}, 70.13 ; \mathrm{H}, 6.20 ; \mathrm{F}, 5.84 ; \mathrm{N}, 12.19 ; \mathrm{O}, 4.92$.

2g 6-(4-chlorophenyl)-4-(4-fluoro-3-methylphenyl)-1,6-dihydropyrimi dine-2-ol

Yield 84\%, m.p. $166^{\circ} \mathrm{C}$, FT-IR (KBr) cm ${ }^{-1}: 983.19$ (C-F); 2924.18 (C-CH Str); 819.23 (C-Cl); 3053.32 (Ar C-H str); 1662.57 (C=N str); 1592 (Ar $\mathrm{C}=\mathrm{C}$ ); 3246.75 (NH str); $3433(\mathrm{OH}):{ }^{1} \mathrm{HNMR}\left(400 \mathrm{MHz}^{\mathrm{CDCl}} \mathrm{CD}_{3} \delta \mathrm{ppm}\right) 2.377$ (s, $\left.3 \mathrm{H}, \mathrm{CH}_{3}\right) ; 1.771(\mathrm{~s}, 1 \mathrm{H}, \mathrm{O}-\mathrm{H}) ; 1.740(\mathrm{~m}, 1 \mathrm{H}, \mathrm{N}-\mathrm{H}) ; 2.188$ (d, J=1.2Hz, $1 \mathrm{H}, \mathrm{C}_{6}-\mathrm{H}$ pyrimidine $) ; 7.150\left(\mathrm{~m}, \mathrm{~J}=8.4 \mathrm{~Hz}, 1 \mathrm{H}, \mathrm{C}_{5}-\mathrm{H}\right.$ pyrimidine $)$; $7.403\left(\mathrm{~m}, 2 \mathrm{H}, \mathrm{C}_{2 n^{\prime \prime}}-\mathrm{H}\right.$ and $\left.\mathrm{C}_{60^{\prime \prime}}-\mathrm{H}\right) ; 7.603\left(\mathrm{~m}, 2 \mathrm{H}, \mathrm{C}_{3^{\prime \prime}}-\mathrm{H}\right.$ and $\left.\mathrm{C}_{5^{\prime \prime}}-\mathrm{H}\right) ; 7.508-$ $7.922\left(\mathrm{~m}, 4 \mathrm{H}, \mathrm{C}_{2}-\mathrm{H}_{1} \mathrm{C}_{5}-\mathrm{H}\right.$ and $\left.\mathrm{C}_{6}-\mathrm{H}\right)$ : MS (EI) m/z: $316\left(\mathrm{M}^{+}\right)$. Anal. calcd for $\mathrm{C}_{17} \mathrm{H}_{14} \mathrm{ClFN}_{2} \mathrm{O} ; \mathrm{C}, 64.46 ; \mathrm{H}, 4.45 ; \mathrm{Cl}, 11.19 ; \mathrm{F}, 6.00 ; \mathrm{N}, 8.84 ; 0,5.05$.

2h 6-(anthracen-9-yl)-4-(4-fluoro-3-methylphenyl)-1,6-dihydropyrimi dine-2-ol

Yield 70\%, m.p. $171^{\circ} \mathrm{C}$, FT-IR (KBr) cm ${ }^{-1}: 1037.27$ (C-F); $2922.80\left(\mathrm{C}-\mathrm{CH}_{3}\right.$ Str); 2854.51 (C-H str) 1658.91 (C=N str); 1455 (Ar C=C); 3439.50 (NH str); $3739(\mathrm{OH}) ;{ }^{1} \mathrm{HNMR}\left(400 \mathrm{MHz}, \mathrm{CDCl}_{3}, \delta \mathrm{ppm}\right) 2.382\left(\mathrm{~s}, 3 \mathrm{H}, \mathrm{CH}_{3}\right)$; 1.622 (s, 1H, O-H); $2.193(\mathrm{~m}, 1 \mathrm{H}, \mathrm{N}-\mathrm{H}) ; 3.518$ (d, J=5.6Hz, 1H, C $6^{-}-\mathrm{H}$ pyrimidine); $7.173\left(\mathrm{~m}, \mathrm{~J}=8.4 \mathrm{~Hz}, 1 \mathrm{H}, \mathrm{C}_{5}-\mathrm{H}\right.$ pyrimidine); 7.527-8.883 (m, 9H, Anthracene-H); 7.999-7.932 (m, 3H, Ar-H): MS (EI) m/z: $382\left(\mathrm{M}^{+}\right)$. Anal. calcd for $\mathrm{C}_{17} \mathrm{H}_{14} \mathrm{ClFN}_{2} \mathrm{O} ; \mathrm{C}, 64.46 ; \mathrm{H}, 4.45 ; \mathrm{Cl}, 11.19 ; \mathrm{F}, 6.00 ; \mathrm{N}, 8.84$; $0,5.05$.

2i 6-(5-bromo-2-hydroxy-3-methoxyphenyl)-4-(4-fluoro-3-methylphe nyl)-1,6-dihydro pyrimidine-2-ol

Yield 73\%, m.p. $160^{\circ} \mathrm{C}$, FT-IR (KBr) cm${ }^{-1}: 1072$ (C-F): 851 (C-Br): 2928 (C-CH ${ }_{3}$ Str): 2926 (C-H str) 1607 (C=N str): 1399 (Ar C=C): 3421 (NH str): $3562(\mathrm{OH}): 1223\left(\mathrm{O}-\mathrm{CH}_{3}\right) ;{ }^{1} \mathrm{HNMR}\left(400 \mathrm{MHz}, \mathrm{CDCl}_{3}, \delta \mathrm{ppm}\right)$ 2.49 (s, 3H, CH $)$; 1.798 (s, $1 \mathrm{H}, \mathrm{N}-\mathrm{H}) ; 2.052(\mathrm{~m}, 1 \mathrm{H}, \mathrm{O}-\mathrm{H}) ; 3.89(3 \mathrm{H}$, $\left.\mathrm{OCH}_{3}\right) ; 4.519\left(\mathrm{~d}, \mathrm{~J}=8.2 \mathrm{~Hz}, 1 \mathrm{H}, \mathrm{C}_{6}-\mathrm{H}\right.$ pyrimidine); $7.69(1 \mathrm{H}, \mathrm{OH}) ; 7.06$ $\left(\mathrm{m}, \mathrm{J}=7.6 \mathrm{~Hz}, 1 \mathrm{H}, \mathrm{C}_{5}-\mathrm{H}\right.$ pyrimidine); $6.91\left(1 \mathrm{H}, \mathrm{C}_{\mu^{\prime \prime}}-\mathrm{H}\right) ; 7.55\left(1 \mathrm{H}, \mathrm{C}_{6^{\prime \prime}}-\mathrm{H}\right)$; $7.22\left(1 \mathrm{H}, \mathrm{C}_{2}-\mathrm{H}\right) ; 7.29\left(1 \mathrm{H}, \mathrm{C}_{5}-\mathrm{H}\right) ; 7.91\left(1 \mathrm{H}, \mathrm{C}_{6}-\mathrm{H}\right): \mathrm{MS}(\mathrm{EI}) \mathrm{m} / \mathrm{z}: 407\left(\mathrm{M}^{+}\right)$. Anal. calcd for $\mathrm{C}_{17} \mathrm{H}_{14} \mathrm{ClFN}_{2} \mathrm{O} ; \mathrm{C}, 53.09 ; \mathrm{H}, 3.96 ; \mathrm{Br}, 19.63 ; \mathrm{F}, 4.67 ; \mathrm{N}, 6.88$; $0,11.79$.

\section{Anti-inflammatory activity}

In vitro anti-inflammatory screening

The human red blood cell (HRBC) membrane stabilization method The blood was collected from a healthy human volunteer and mixed with equal volume of Alsever solution ( $2 \%$ dextrose, $0.8 \%$ sodium citrate, $0.5 \%$ citric acid, and $0.42 \% \mathrm{NaCl}$ ) and centrifuged at $3000 \mathrm{rpm}$ for $10 \mathrm{~min}$. The packed cells were washed with iso-saline $(0.36 \%)$ and a $10 \%$ suspension was made. Various concentrations of 4-(4-fluoro-3-methylphenyl)-6(substituted aryl)-1,6-dihydropyrimidin-2-ol (2a-2j) were prepared $(75,150$, and $200 \mu \mathrm{g} / \mathrm{ml})$ using distilled water and to each concentration $1 \mathrm{ml}$ of phosphate buffer, $2 \mathrm{ml}$ hyposaline, and $0.5 \mathrm{ml}$ of $\mathrm{HRBC}$ suspension were added. It was incubated at $37^{\circ} \mathrm{C}$ for $30 \mathrm{~min}$ and centrifuged at $3000 \mathrm{rpm}$ for $20 \mathrm{~min}$, and the hemoglobin content of the supernatant solution was estimated spectrophotometrically at $560 \mathrm{~nm}$. Diclofenac $(75,150$, and $200 \mu \mathrm{g} / \mathrm{ml})$ was used as the reference standard, and the control was prepared by omitting the compounds under examination.

The percentage of HRBC membrane stabilization or protection was calculated using the following formula:

$$
\% \text { Membranestabilization }=100-\frac{\text { O.D Of Test }}{\text { O.D Of Control }} \times 100
$$

\section{Ethical approval}

All experiments have been examined and approved by the Institutional Animal Ethics Committee at the GITAM University, Visakhapatnam, India (Approved proposal No:- IAEC/GIP-1287/CAD-UGC/ approved/3/2015). Animal experiments were also performed in accordance with the Guidelines on Ethical Standards for Investigation of Experimental Pain in Animals [18].

\section{In vivo anti-inflammatory activity}

Wistar albino rats of either sex (200 - 250 gms) were procured from Ghosh Enterprises kolkatta, West Bengal. A total of 72 rats were divided into 12 groups of 6 rats each. They were allowed for fasting overnight and given water ad libitum. Group I was given only $1 \%$ sodium carboxymethyl cellulose suspension $(1 \mathrm{ml} / \mathrm{kg})$ and was used as carrageenan-treated control. Group II was treated with the standard drug diclofenac $(100 \mathrm{mg} / \mathrm{kg})$. Similarly, the rest of the groups were administered with test drugs $(2 \mathrm{a}-2 \mathrm{i})$, respectively. The test drugs (100 mg/kg body weight) and the standard drugs (100 mg/kg body 
weight) were administered orally with the help of the oral catheter. After $30 \mathrm{~min}, 0.05 \mathrm{ml}$ of $1 \%$ carrageenan suspension was slowly injected subcutaneously into the subplantar region of the left hind paw to all the groups to produce inflammation. After the administration of carrageenan, the volume of its displacement was measured volumetrically by comparing with 0 min reading and again after every $1,2,3$, and $4 \mathrm{~h}$ of induction with plethysmometer apparatus and compared. The percentage increase of paw thickness was determined at $0,1,2,3$, and $4 \mathrm{~h}$ after induction of inflammation.

The anti-inflammatory activity was expressed as percentage inhibition.

$$
\% \text { Inhibition }=\frac{\text { Control }- \text { test }}{\text { Control }} \times 100
$$

\section{Analgesic activity}

The acetic acid writhing test was performed on Wistar albino rats by following the method of Berkowitz et al. [19]. Test compounds were given to the animals at the dose of $50 \mathrm{mg} / \mathrm{kg}, 30 \mathrm{~min}$ later the animals have injected intraperitoneally with $0.25 \mathrm{ml} / \mathrm{rat}$ of $0.5 \%$ acetic acid. The mean number of writhes for each experimental groups and the percentage decrease compared with the control group was calculated after $60 \mathrm{~min}$.

\section{RESULTS AND DISCUSSION}

The title compounds $2 \mathrm{a}-2 \mathrm{i}$ were synthesized as per the protocol shown in Scheme 1. In the present work, by substituting different aryl moiety at the C-6 position of 4-(4-fluoro-3-methylphenyl)-1,6-dihydropyrimidin2 -ol, a sequence of novel pyrimidine derivatives $2 \mathrm{a}-2 \mathrm{i}$ was synthesized. The presence of particular groups was identified from IR spectra by means of some characteristic absorption bands. The IR spectrum of chalcones showed characteristic intense absorption bands at 1657 ( $\mathrm{C}=\mathrm{O}$, chalcone), 1585 ( $\mathrm{C}=\mathrm{C}), 1149$ (C-Fl), and $2949\left(\mathrm{C}-\mathrm{CH}_{3}\right)$. The formation of pyrimidine was confirmed from the absorption bands of IR spectra. The absorption band at 3449.10 indicates NH Stretch of the pyrimidine ring. Further, it can also be confirmed from the ${ }^{1} \mathrm{H}$ NMR spectral data. A strong peak at $\delta 1.593$ ppm integrating for $\mathrm{N}-\mathrm{H}$ proton of pyrimidines. The spectrum also revealed a doublet at $\delta 2.194 \mathrm{ppm}$ for the proton of $\mathrm{C}-6-\mathrm{H}$ of the pyrimidine ring. A singlet peak at $\delta 2.34 \mathrm{ppm}$ for three protons which might be assigned to $\mathrm{CH}_{3}$. The structure of title compounds $2 \mathrm{a}-2 \mathrm{i}$ was further confirmed by the appearance of various other peaks in NMR spectroscopy corresponding to the assigned structure. In addition, the data of the mass spectrum further confirmed their molecular weight and purity.

\section{Biological activities}

In vitro anti-inflammatory activity (HRBC membrane stabilization method)

In vitro anti-inflammatory activity of test compounds was evaluated using the HRBC membrane stabilization method. The anti-inflammatory activity results (Table 1 ) revealed that all the test compounds showed better activity when compared to that of standard drug. From the results, it was observed that the compounds $2 \mathrm{~g}$ and $2 \mathrm{~b}$ exhibited good activity when compared to that of standard drug. It may be due to the presence of halogen group on the phenyl ring attached at position- 6 of pyrimidine. The compound $2 \mathrm{i}$ also showed good activity. The presence of bulk anthracene group attached to the pyrimidine ring may contribute to its activity. The compound $2 \mathrm{a}$ with thiophene moiety attached to the pyrimidine ring also showed good activity. Rest of the compounds showed moderate anti-inflammatory activity.

\section{In vivo anti-inflammatory activity (carrageenan-induced rat paw} edema method)

In vivo anti-inflammatory activity of test compounds was evaluated using carrageenan-induced rat paw edema method. The anti-inflammatory activity results (Table 2 ) revealed that all the test compounds showed better activity when compared to that of standard drug. The phenyl ring substituted with hydroxyl, methoxy, and nitro substituents attached at position- 6 of pyrimidine causes a decrease in the activity of the compound $2 \mathrm{~d}$. The compound 2e possessing dimethoxyphenyl ring at position- 6 of pyrimidine ring exhibited moderate anti-inflammatory activity when compared to the reference standard diclofenac sodium. Replacement of dimethoxy phenyl ring with 5-bromo-2-hydroxy3-methoxy phenyl ring $2 \mathrm{i}$ leads to an increase in the activity. With increased lipophilicity, the compound with dimethylamino substituent $2 \mathrm{f}$ and nitrophenyl substituents $2 \mathrm{c}$ showed the least activity. Among all tested compounds para chloro analog $2 \mathrm{~g}$ exhibited a better activity which was more potent than diclofenac. Compounds with anthracene moiety $2 \mathrm{~h}, 2$-bromo phenyl $2 \mathrm{~b}$ and thiophene ring $2 \mathrm{a}$ also showed better activity.

\section{Analgesic activity}

Entire test compounds $2 \mathrm{a}-2 \mathrm{i}$ were tested for their analgesic activity by the tail-flick technique using Wistar albino mice. The results of the analgesic study were summarized in Table 2 . Compounds $2 \mathrm{~g}$ with 4-chlorophenyl derivative and $2 \mathrm{~b}$ with 2-bromophenyl derivative showed similar analgesic activity compared to standard drug diclofenac sodium. Replacement of chlorine group with nitro or dimethylamino or methoxy or hydroxyl groups results in a sharp fall in the activity. It may be due to a decrease in the lipophilicity. From the results, it was found that the pyrimidine derivatives with halogen substituents showed better activity when compared to other derivatives. Compounds 6-(4-chlorophenyl)-4-(4-fluoro-3-methylphenyl)-1,6dihydropyrimidin-2-ol 2g and 4-(4-fluoro -3-methylphenyl)-6-(2bromo phenyl)-1,6-dihydropyrimidin-2-ol $2 \mathrm{~b}$ were found to be the most active analgesic agent and it showed similar potency when compared to the reference standard diclofenac sodium.

\section{CONCLUSION}

In summary, a series of novel pyrimidine derivatives $2 \mathrm{a}-2 \mathrm{i}$ were synthesized and characterized by FT-IR, ${ }^{1} \mathrm{H}-\mathrm{NMR}$, mass spectroscopy, and elemental analysis. These derivatives were evaluated for their analgesic

Table 1. Anti-inflammatory activity of pyrimidine (2a-2i) HRBC method

\begin{tabular}{|c|c|c|c|}
\hline S.No & Compound & Concentration $(\mu \mathrm{g} / \mathrm{ml})$ & \%Stabilization \\
\hline \multirow[t]{3}{*}{1.} & $2 \mathrm{a}$ & 75 & 50.21 \\
\hline & & 150 & 69.12 \\
\hline & & 200 & 80.16 \\
\hline \multirow[t]{3}{*}{2.} & $2 b$ & 75 & 49.84 \\
\hline & & 150 & 58.12 \\
\hline & & 200 & 82.56 \\
\hline \multirow[t]{3}{*}{3.} & $2 c$ & 75 & 39.06 \\
\hline & & 150 & 55.15 \\
\hline & & 200 & 71.46 \\
\hline \multirow[t]{3}{*}{4.} & $2 \mathrm{~d}$ & 75 & 41.65 \\
\hline & & 150 & 55.51 \\
\hline & & 200 & 65.23 \\
\hline \multirow[t]{3}{*}{5.} & $2 \mathrm{e}$ & 75 & 44.61 \\
\hline & & 150 & 56.18 \\
\hline & & 200 & 76.35 \\
\hline \multirow[t]{3}{*}{6.} & $2 \mathrm{f}$ & 75 & 46.54 \\
\hline & & 150 & 54.21 \\
\hline & & 200 & 64.11 \\
\hline \multirow[t]{3}{*}{7.} & $2 \mathrm{~g}$ & 75 & 51.17 \\
\hline & & 150 & 64.25 \\
\hline & & 200 & 91.12 \\
\hline \multirow[t]{3}{*}{8.} & $2 \mathrm{~h}$ & 75 & 46.93 \\
\hline & & 150 & 61.62 \\
\hline & & 200 & 74.45 \\
\hline \multirow[t]{3}{*}{9.} & $2 \mathrm{i}$ & 75 & 49.44 \\
\hline & & 150 & 66.16 \\
\hline & & 200 & 88.61 \\
\hline \multirow[t]{3}{*}{10.} & Diclofenac & 75 & 64.26 \\
\hline & & 150 & 78.74 \\
\hline & & 200 & 94.67 \\
\hline
\end{tabular}

HRBC: Human red blood cell 


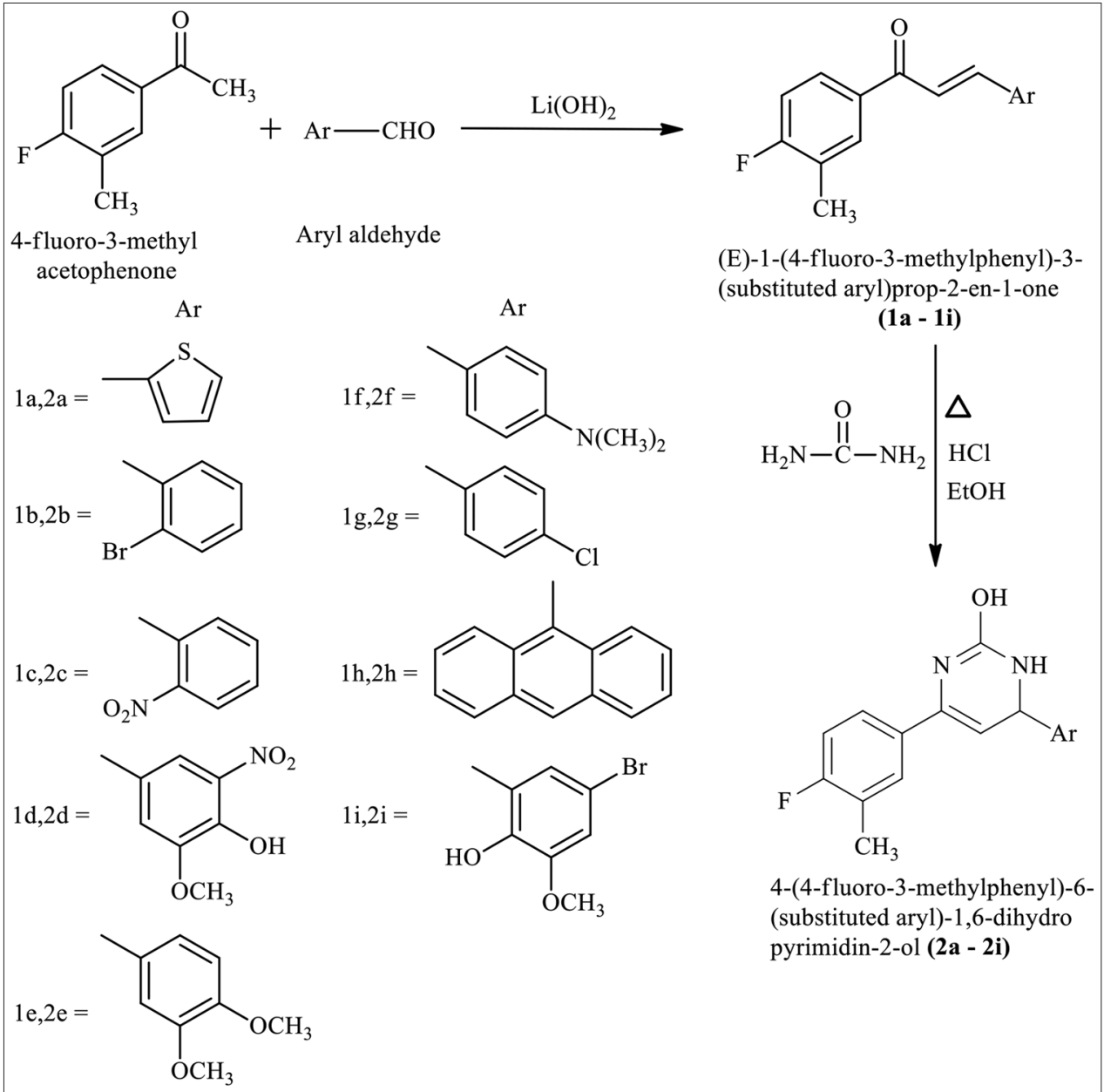

Scheme 1: Synthesis of 4-(4-fluoro-3-methylphenyl)-6-(substituted aryl)-1,6-dihydropyrimidin-2-ol (2a -2i)

Table 2: Effect of pyrimidine derivatives in carrageenan-induced paw edema in rats and analgesic activity

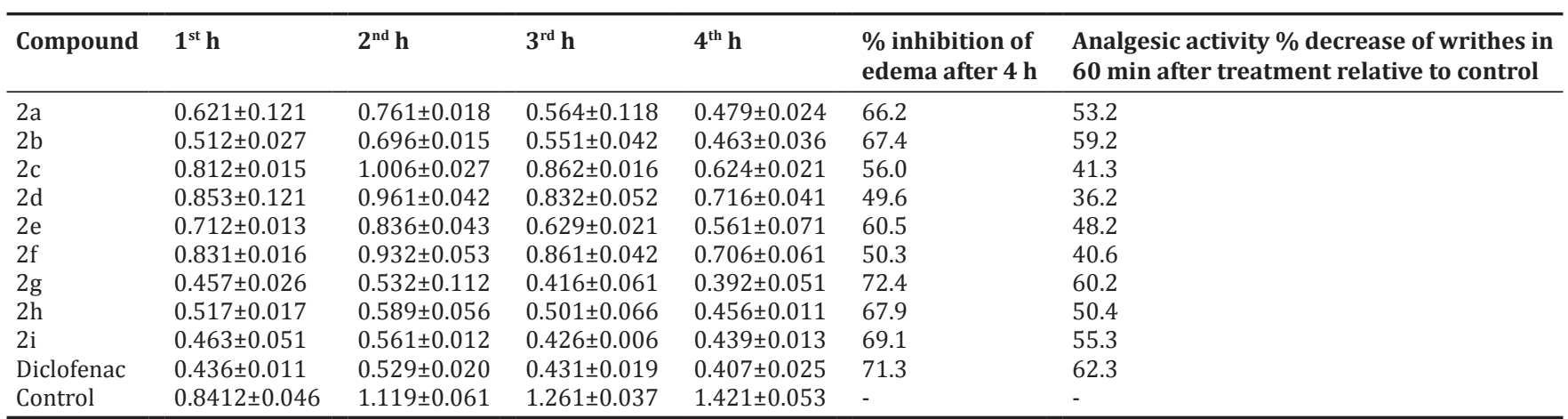

and anti-inflammatory activities. In general, chlorine substituted compounds exhibited potent analgesic and anti-inflammatory activities. From the study, it was concluded thatin this series nature of the substituent played a major role in analgesic and anti-inflammatory activity than its position. Among several tested compounds, 6-(4-chlorophenyl)-4-(4fluoro-3-methylphenyl)-1,6-dihydro pyrimidin-2-ol $2 \mathrm{~g}$ showed better analgesic and anti-inflammatory activities which were more potent than reference standard diclofenac. Hence, this analog could be developed as a new class of analgesic and anti-inflammatory agent.

\section{ACKNOWLEDGMENT}

The authors are thankful to the UGC (New Delhi, India) for providing financial assistance to carry out the research work.

\section{AUTHOR'S CONTRIBUTIONS}

Muralidharan V: Performed the experiments. Dr. C. Asha Deepti: Conceived the idea, study design and finalized the manuscript. Dr. S. Raja: Assisted in experimental work and helped in the preparation of the manuscript.

\section{CONFLICTS OF INTEREST}

There are no conflicts of interest.

\section{REFERENCES}

1. Yazdan SK, Sagar DV, Shaik AB. Synthesis, characterization and biological evaluation of some new pyrimidine derivatives as anti- 
inflammatory and cytotoxic agents. Res J Pharm Biol Chem Sci 2015;6:173-85.

2. Nigam SC, Saharia GS Sharma HR. Studies in heterocyclic compounds. Part 38: Synthesis and in vitro screening of 4, 6-dimethyl-5-(arylazo/ Arsubstituted p-sulfamoylbenzeneazo) pyrimidine-2-thiols. Indian Chem Soc 1983;60:583-4.

3. Liang Y, Wnuk SF. Modification of purine and pyrimidine nucleosides by direct C-H bond activation. Molecules 2015;20:4874-901.

4. Rao NV, Vaisalini NB, Mounika B, Harika VL, Kumar DP, Sreekanth N. Review article an overview on synthesis and biological activity of pyrimidines. Int J Pharm Chem Res 2013;2:2278-87.

5. Kumar B. Pyrimidines as potent cytotoxic and anti-inflammatory agents. Asian J Pharm Clin Res 2017;10:237-9.

6. Subbraju GV, Nayakulu AR, Parameshwara D. Pyrazoline enaminessynthesis and characterization of some conjugated enamino esters derived from 3-aroyl-4-aryl-2-pyrazolines. Indian J Heterocycl Chem 1994:4:87-92.

7. Jainey PJ, Ishwar BK. Microwave assisted synthesis of novel pyrimidines bearing benzene sulfonamides and evaluation of anticancer and antioxidant activities. Asian J Pharm Clin Res 2014;7:111-4.

8. Liu J, Wu F, Chen L, Hu J, Zhao L, Chen C, et al. Evaluation of dihydropyrimidin- $(2 \mathrm{H})$-one analogues and rhodanine derivatives as tyrosinase inhibitors. Bioorg Med Chem Lett 2011;21:2376-9.

9. Nofal ZM, Fahmy HH, Zarea ES, El-Eraky W. Synthesis of new pyrimidine derivatives with evaluation of their anti-inflammatory and analgesic activities. Acta Pol Pharm 2011;68:507-17.

10. Manisha SP, Gourishankar RA. Molecular docking study on $1 \mathrm{~h}-(3,4 \mathrm{~d})$ pyrazolo-pyrimidines as cyclin dependant kinase (cdk2) inhibitors. Int
J Curr Pharm Res 2017 9:94-100.

11. Vitthal AD, Dhongade-desai S. An efficient microwave assisted multi-component synthesis of some 7-amino-3-(substituted phenyl)5-(substituted phenyl)-[1, 2, 4]triazolo[4,3a]pyrimidine-6-carbonitrile derivatives. Int J Curr Pharm Res 2014;6:20-4.

12. Cieplik J, Stolarczyk M, Pluta J, Gubrynowicz O, Bryndal I, Lis T, et al. Synthesis and antibacterial properties of pyrimidine derivatives. Acta Pol Pharm 2011;68:57-65.

13. Gilchrist TL. Heterocyclic Chemistry. UK: Longman Scientific and Technical Publisher; 1984. p. 187.

14. Wermuth CG. The Practice of Medicinal Chemistry. New York: Academic Press; 1996. p.257.

15. Bhandari SV, Dangre SC, Bothara KG, Patil AA, Sarkate AP, Lokwani DK. Design, synthesis and pharmacological screening of novel nitric oxide donors containing 1,5-diarylpyrazolin-3-one as nontoxic NSAIDs. Eur J Med Chem 2009;44:4622-36.

16. Olfert ED, Cross BM, McWilliam AA. Canadian Council of Animal Care Guide to the Care and use of Experimental Animals. Vol. 1. $2^{\text {nd }}$ ed. Ottawa; Canadian Council on Animal Care: 1993

17. Boeck P, Falcão CA, Leal PC, Yunes RA, Filho VC, Torres-Santos EC, et al. Synthesis of chalcone analogues with increased antileishmanial activity. Bioorg Med Chem 2006;14:1538-45.

18. Zimmermann M. Ethical guidelines for investigations of experimental pain in conscious animals. Pain 1983;16:109-10.

19. Berkowitz BA, Finck SA, Ngai SH. Nitrous oxide analgesia: Reversal by naloxone and development of tolerance. J Pharmacol Exp Ther 1977; 203:539-47. 